\title{
Electric-field-induced optical second-harmonic generation in poly(phenylene vinylene) light-emitting diodes
}

\author{
R. Hildebrandt, H.-M. Keller, G. Marowsky \\ Laser-Laboratorium Göttingen e.V., Hans-Adolf-Krebs-Weg 1, 37077 Göttingen, Germany \\ W. Brütting, T. Fehn, M. Schwoerer \\ Lehrstuhl für Experimentalphysik II, Universitätsstrasse 30, 95440 Bayreuth, Germany \\ J. E. Sipe \\ Department of Physics, University of Toronto, 60 St. George Street, Toronto, Canada
}

\begin{abstract}
We demonstrate second-harmonic generation in polymeric light-emittingdiodes built in a layer structure of indium tin oxide, poly(phenylene vinylene) and aluminum. The second-harmonic intensity generated in the active zone of the diode depends quadratically on the applied reverse bias. In order to account for the bias dependence of the measured intensity a simple model is given in that the signal consists of two contributions: a constant, bias independent contribution from the indium tin oxide / poly(phenylene vinylene) interface and a second one that depends on the effective bias at the organic metal-semiconductor contact. Further applications for second-harmonic spectroscopy in organic semiconductors are discussed.
\end{abstract}


Due to its high intrinsic surface sensitivity second-harmonic generation (SHG) has been established as a very powerful tool for the analysis of surfaces and interfaces. Especially in the field of metal oxide semiconductor interfaces various investigations have been performed recently. Within this framework the influences of external static or high-frequency electric fields on the SH intensity were extensively investigated [1-3]. These electric-field-induced second-harmonic (EFISH) measurements allow an in situ determination of important quantities in electrical devices or high-frequency circuits with very high time-resolution up to the GHz-domain. All these investigations dealt with inorganic semiconductor materials. The purpose of this paper is to demonstrate that the EFISH technique can also be applied to organic semiconductors like the poly(phenylene vinylene) (PPV); a commonly used material for the fabrication of organic light-emitting-diodes, LEDs.

Diodes based on PPV are extensively studied due to possible applications in displaytechnology. We consider EFISHG for PPV diodes in particular being interesting since SHG in PPV is forbidden by symmetry. In contrast to thermal poling experiments [4] the EFISHG technique allow an in situ investigation at normal operating temperature and leads to no permanent nonlinear susceptibilty.

The LEDs used for our experiments are built up in a layer structure as shown in Fig. 1, consisting of glass as substrate, a $30 \mathrm{~nm}$ indium tin oxide (ITO) layer as transparent electrode, a $300 \mathrm{~nm}$ poly(phenylene vinylene) layer and an aluminum top electrode; details of the fabrication processes are published elsewhere [5]. Since the ITO and the aluminum layer are structured in a stripe pattern perpendicular to each other, the active zone of the diode $(\mathrm{ITO} / \mathrm{PPV} / \mathrm{Al})$ is the resultant square where the two stripes overlap. The samples thus have various regions where the individual interfaces can be investigated by SHG seperately. For the measurements the sample was attached with its glass side onto a prism using glycerin as immersion. All measurements were performed under normal incidence on the prism. The laser setup consists of a Nd:YAG laser (8 ns, $10 \mathrm{~mJ}, 1064 \mathrm{~nm}, 10 \mathrm{~Hz}$ ) and a standard monochromator/photomultiplier combination for the SH detection (details cf. [6]). A polarizer and an analyzer allowed polarization dependent excitation and detection. In order to 
prevent light induced damage to the diode the pulse energy of the fundamental was kept below $5 \mathrm{~mJ}$ at a beam diameter of about $2 \mathrm{~mm}$. To perform bias dependent $\mathrm{SH}$ measurements in the active zone of the diode, the electrodes of the sample were connected to a computer controlled power supply. In order to prevent degeneration of the diode, measurements were performed with an maximum reverse bias of $20 \mathrm{~V}$ and a forward bias not exceeding $2 \mathrm{~V}$.

SH measurements at different positions on the sample allow to seperate the contributions of the various interfaces to the SH intensity. For these measurements both excitation and detection were performed in p-polarization without applying any bias to the diode. PPV on glass shows no significant SH Intensity corresponding to the expectations for an centrosymmetric material, whereas in the ITO/PPV, the $\mathrm{PPV} / \mathrm{Al}$ and the ITO/PPV/Al region a signal is observed. The measured signal was successfully identified as SH by its spectral properties and its intensity dependence.

The signal in the active zone of the diode is shown in Fig. 2. Even if no bias is applied, a SH signal is detected. With increasing reverse bias, the SH intensity first decreases to a minimum value and after that it increases quadratically. For a maximum voltage of $20 \mathrm{~V}$, the SH-intensity is twenty-five times larger than that of the unbiased diode.

In order to account for that bias dependence we present the following phenomenological model. At first, we try to give an explanation for the fact that there is a bias dependent signal at all. The physical properties of the PPV/Al-contact can be well described by that of a Schottky contact [?]. At the contact, there is a depletion region exhibiting the bias dependent thickness $d$. This thickness is similiar to the square root of the effective bias [7],

$$
d \propto \sqrt{U_{\text {eff }}} \propto \sqrt{U_{\mathrm{D}}-U}
$$

where $\mathrm{U}$ denotes the external applied bias and $U_{\mathrm{D}} \approx 1.8 \mathrm{~V}$ is the diffusion voltage which is defined as the potential difference over the Schottky contact in the unbiased case.

In the depletion region, a polarization is induced in PPV by the corresponding electric field $E_{0}(z)$, hence the centrosymmectric symmetry is broken and a signal can be detected. The electric-field-induced second-harmonic generation can be described by the third order 
susceptibility $\chi^{(3)}=\chi^{(3)}(-2 \omega ; \omega ; \omega ; 0)$; supposing that phase-matching can be neglected, the SH intensity $I_{2 \omega}$ for $U \leq U_{\mathrm{D}}$ is given by

$$
\begin{aligned}
I_{2 \omega} & \propto\left|\int_{0}^{d} \chi^{(3)}(-2 \omega ; \omega ; \omega ; 0) E_{\omega}^{2} E_{0}(z) d z\right|^{2} \\
& \propto\left|\chi^{(3)} I_{\omega} \int_{0}^{d} E_{0}(z) d z\right|^{2} \\
& \propto\left|\chi^{(3)}\left(U_{\mathrm{D}}-U\right)\right|^{2} I_{\omega}^{2} .
\end{aligned}
$$

With Eq. (4) we exspect a branch of a parabola shifted to the right by the diffusion voltage $U_{\mathrm{D}}$. However, Fig. 2 show a minimum shifted to the left, therefore a constant, bias independent $\chi_{\text {const }}^{(2)}$ contribution to the SH signal has to be assumed. As illustrated in Fig. 3, the total signal is determined by the complex addition of these two contributions. With $\chi_{\text {eff }}^{(2)}(U)=\chi^{(3)}\left(U_{\mathrm{D}}-U\right)$ and $\Delta \phi$ being the phasedifference of the two contributions, the SH intensity can be calculated as

$$
\begin{aligned}
I_{2 \omega} & \propto\left|\chi_{\text {eff }}^{(2)}(U)+\chi_{\text {const }}^{(2)}\right|^{2} I_{\omega}^{2} \\
& \propto\left(\left|\chi^{(3)}\right|^{2}\left(U_{\mathrm{D}}-U\right)^{2}+\left|\chi_{\text {const }}^{(2)}\right|^{2}+2\left|\chi_{\text {const }}^{(2)}\right|\left|\chi^{(3)}\right|\left(U_{\mathrm{D}}-U\right) \cos \Delta \varphi\right) I_{\omega}^{2} .
\end{aligned}
$$

Using this equation, the experimental data can be fitted quite well as shown by the black line in Fig. 2. We assume that the constant contribution results from the ITO/PPV interface as was varified by a reference measurement in the ITO/PPV region of the sample which give a signal of similar magnitude. The strong SH signal of the ITO/PPV interface is well known in literature [8].

Figure 4 shows the build-up of the $\mathrm{SH}$ on a microsecond time-scale after switching on a reverse bias of $U_{0}=21.5 \mathrm{~V}$ using a sampling technique where the time delay between the rising edge of the bias and the laser pulse was varied as depicted in the inset of Fig. 4. The measured time-dependence reflects the exponential increase of $U(t)$ at the diode that acts as a capacitor where the SH signal depends quadratically on $U(t)$ according to Eq. (6). Besides, the experimental data prove that the bias dependence is reproducable and only due to reversible electric-field-induced processes. In contrast to thermal poling experiments [4] 
no permanent effect remains after the bias is switched off. Time-resolved measurements may be helpful to determine electrical properties especially for ultrahigh time-resolution that can be reached by fs-pulses.

We also investigated the bias dependence of the second-harmonic in forward direction. Since our setup had no encapsulation to protect the diode from degeneration during operation, the response of the diode changed dramatically during operation. For that reason we were not able to find out a reproducable correlation of bias, current and SH intensity. However, in the case that the diode does not allow any current in forward direction due to the build-up of a high resistant layer, a strong bias dependent signal could be observed even in forward direction. Thus the bias dependence in forward direction may give the possibility to observe the build-up of such a layer in situ and thus might be important for understanding the processes leading to the degeneration of the diode.

In summary, we showed that it is possible to apply the EFISHG technique to anorganic semiconductor devices. The ITO/PPV/Al-diode shows a quadratic dependence from the applied bias that can be explained by simple model. The EFISHG technique may be helpful for the in situ investigation of electrical properties with high time resolution and may be of possible use to investigate the ageing of the diodes.

The authors thank J. Jethwa and H.-M. Keller for helpful comments. 


\section{REFERENCES}

[1] Aktisipetrov, A. A. Fedyanin, E. D. Mishina, A. N. Rubtsov, C. W. van Hasselt, M. A. C. Devillers, Th. Rasing, Phys. Rev. B 54, 3 (1996).

[2] G. Lüpke, C. Meyer, C. Ohlhoff, H. Kurz, S. Lehmann, G. Marowsky, Opt. Lett. 20, 1997 (1995).

[3] C. Ohlhoff, G. Lüpke, C. Meyer, H. Kurz, Phys. Rev. B ?, ? (1997).

[4] R. Blum, M. Sprave, J. Sablotny, M. Eich, J. Opt. Soc. Am. B 15, 318 (1998).

[5] M. Herold, J. Gmeiner, C. Drummer, M. Schoerer, J. Mat. Sci. 32, 5709 (1997).

[6] R. Stolle, T. Dürr, G. Marowsky, J. Opt. Soc. Am. B 14, 1583 (1997).

[7] S. M. Sze, Physics of Semiconductor Devices (Wiley \& Sons, New York, 1981).

[8] F. Ghebremichael, C. Poga, M. G. Kuzyk, Appl. Phys. Lett. 66, 139 (1995). 


\section{FIGURES}

FIG. 1. Set-up of the ITO/PPV/Al light-emitting diode.

FIG. 2. Dependence of the second-harmonic intensity on the applied voltage. Negative values imply reverse bias. The plot shows a quadratic dependence of the SH-intensity from the applied voltage. The black line is a fit to the data based on equation 4 with $U_{\mathrm{D}}=1.8 \mathrm{~V}, \Delta \varphi=139.3(21)^{\circ}$, $\chi_{\text {const }}^{(2)}=4.35(14)$ a.u. and $\chi^{(3)}=0.769(14)$ a.u.

FIG. 3. The total second-harmonic intensity is determined by the complex addition of a con-

stant contribution $\chi_{\text {const }}^{(2)}$ and a second part $\chi_{\text {eff }}^{(2)}(U)$ that depends on the effective bias applied to the diode.

FIG. 4. Build-up of the second-harmonic after applying on the reverse bias. The behavior is characterized by the loading process of a capacitor. The black line is a fit to the data decribed in text with $U_{\mathrm{D}}=1.8 \mathrm{~V}, \Delta \varphi=139.9(32)^{\circ}, \chi_{\text {const }}^{(2)}=4.42(22)$ a.u. and $\chi^{(3)}=0.742(3)$ a.u. - Inset: Schematic diagram of the sampling technique for the implementation of time-resolved measurements 


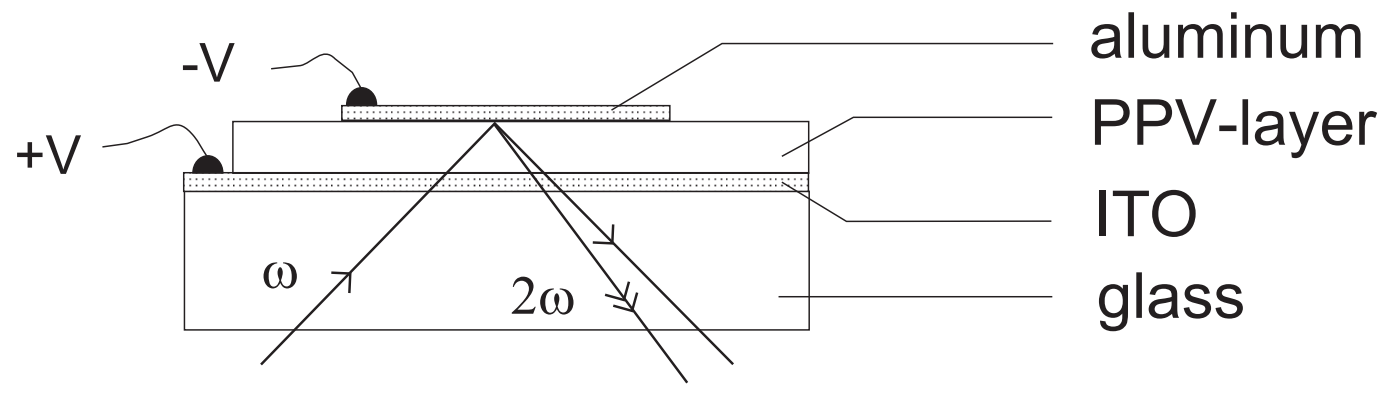

Figure 1

R. Hildebrandt et al.

Optics Letters 


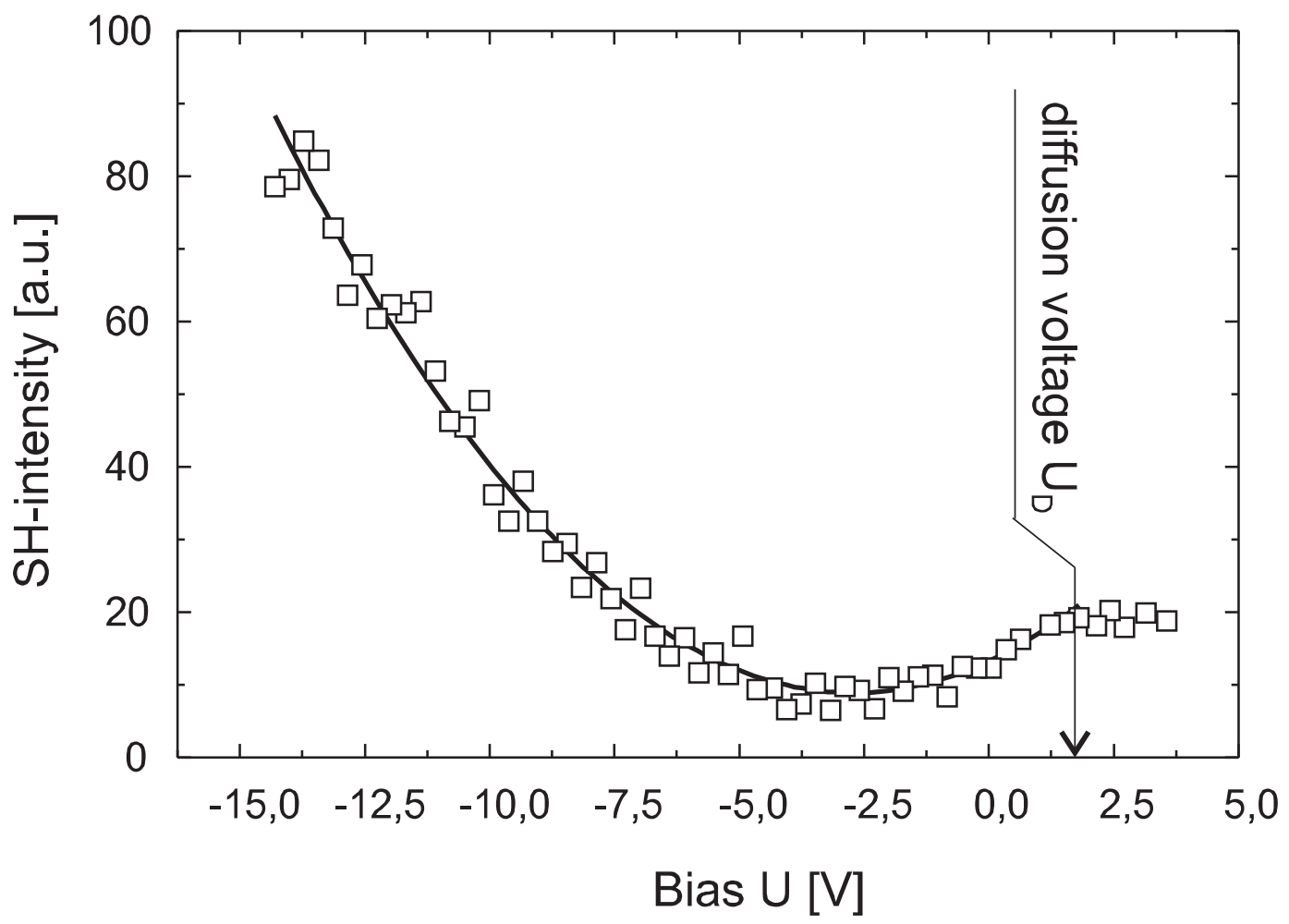

Figure 2

R. Hildebrandt et al.

Optics Letters 


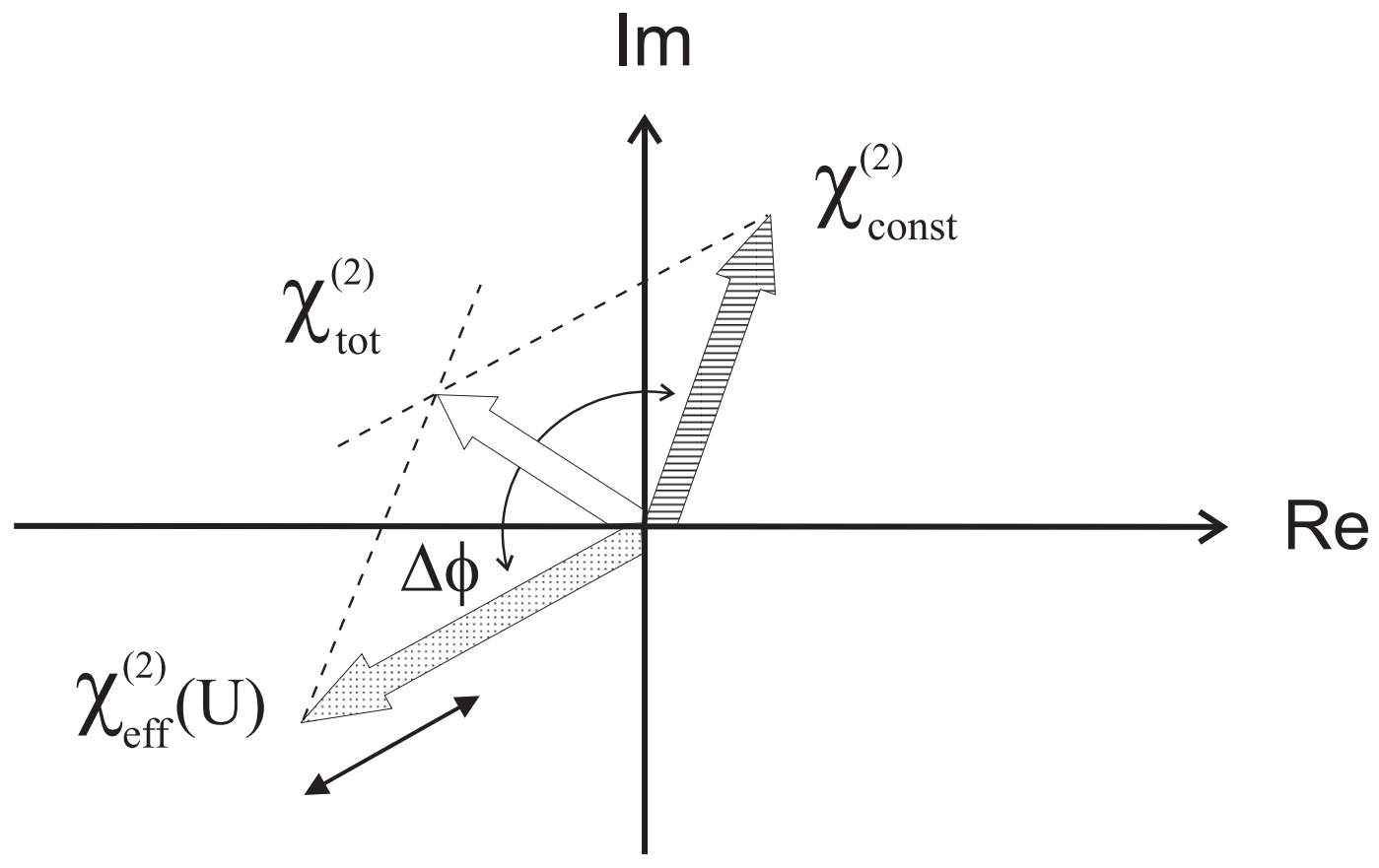

Figure 3

R. Hildebrandt et al.

Optics Letters 


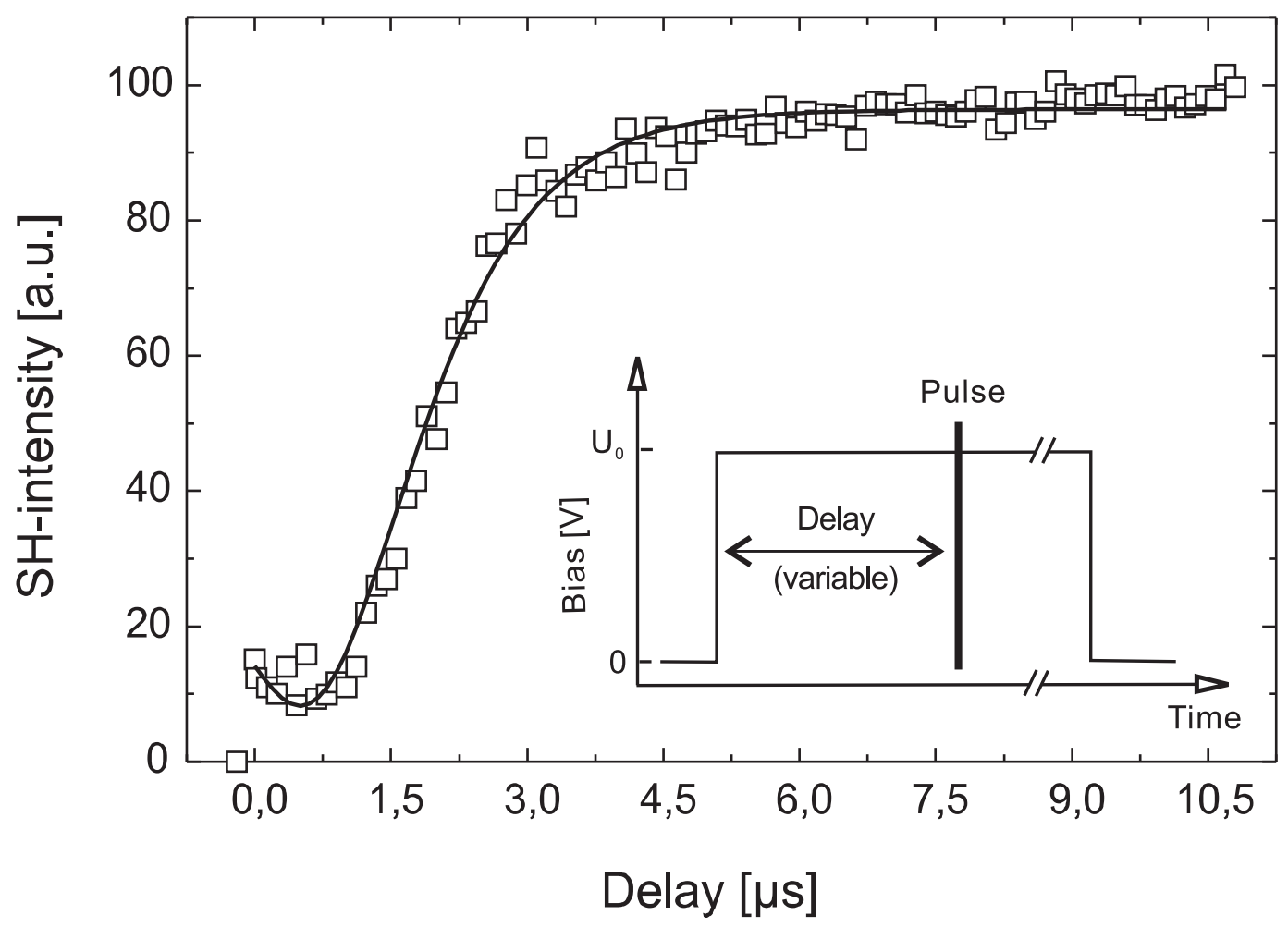

Figure 4

R. Hildebrandt et al.

Optics Letters 